\title{
ARTICLES
}

\section{Stimulation, Monitoring, and Analysis of Pathway Dynamics by Metabolic Profiling in the Aromatic Amino Acid Pathway}

\author{
M. Oldiges, ${ }^{\dagger}$ M. Kunze,${ }^{\dagger}$ D. Degenring, ${ }^{\dagger}$ G. A. Sprenger, ${ }^{\ddagger}$ and R. Takors ${ }^{*}, \dagger$
}

Institute of Biotechnology, Forschungszentrum Jülich GmbH, 52425 Jülich, Germany, and Institute of Microbiology, Universität Stuttgart, Allmandring 31, 70569 Stuttgart, Germany

Using a concerted approach of biochemical standard preparation, analytical access via LC-MS/MS, glucose pulse, metabolic profiling, and statistical data analysis, the metabolism dynamics in the aromatic amino acid pathway has been stimulated, monitored, and analyzed in different tyrosine-auxotrophic L-phenylalanine-producing Escherichia coli strains. During the observation window from -4 s (before) up to 27 $\mathrm{s}$ after the glucose pulse, the dynamics of the first five enzymatic reactions in the aromatic amino acid pathway was observed by measuring intracellular concentrations of 3-deoxy-D-arabino-heptulosonate 7-phosphate $\mathrm{DAH}(\mathrm{P})$, 3-dehydroquinate (3-DHQ), 3-dehydroshikimate (3-DHS), shikimate 3-phosphate (S3P), and shikimate (SHI), together with the pathway precursors phosphoenolpyruvate (PEP) and P5P, the lumped pentose phosphate pool as an alternative to the nondetectable erythrose 4-phosphate (E4P). Provided that a sufficient fortification of the carbon flux into the pathway of interest is ensured, respective metabolism dynamics can be observed. On the basis of the intracellular pool measurements, the standardized pool velocities were calculated, and a simple, data-driven criterion-called "pool efflux capacity" (PEC)-is derived. Despite its simplifying system description, the criterion managed to identify the wellknown AroB limitation in the $E$. coli strain A (genotype $\Delta$ (pheA tyrA aroF)/pJF119EH aro $F^{f b r} p h e A^{f b r} a m p$ ) and it also succeeded to identify AroL and AroA (in strain B, genotype $\Delta$ (pheA tyrA aroF)/pJF119EH aroFfbr pheAfbr aroB amp) as promising metabolic engineering targets to alleviate respective flux control in subsequent L-Phe producing strains. Furthermore, using of a simple correlation analysis, the reconstruction of the metabolite sequence of the observed pathway was enabled. The results underline the necessity to extend the focus of glucose pulse experiments by studying not only the central metabolism but also anabolic pathways.

\section{Introduction}

In their well-known, pioneering work, Theobald et al. (1997) and Rizzi et al. (1997) presented the results of glucose pulse experiments with Saccharomyces cerevisiae to stimulate the yeast metabolism. As shown, sudden changes of glucose availability caused intracellular metabolism dynamics, which has been the basis for identifying in vivo enzyme kinetics by a sequential procedure of glucose pulsing, rapid cell sampling, metabolism inactivation, intracellular metabolite analysis, and detailed modeling using structured, mechanistic models. In the following, similar (experimental) studies have been performed analyzing S. cerevisiae (Vaseghi et al., 1999; Mauch et al., 2000; Lange et al., 2001, Ostergaard et al., 2001), Zymomonas mobilis (Weuster-Botz and de Graaf, 1996; Weuster-Botz D., 1997;) and Escherichia coli

* Corresponding author. Tel: +49/2461/613365. Fax: +49/2461/

613870. E-mail: r.takors@fz-juelich.de.

$\dagger$ Forschungszentrum Jülich GmbH.

¥Universität Stuttgart.
(Schäfer et al., 1999; Sauter et al., 2002; Chassagnole et al., 2002; Hurlebaus et al., 2002; Degenring et al. 2003).

All these studies have in common that they have focused on the analysis of central metabolism dynamics, namely glucose uptake, glycolysis, pentose phosphate pathway, and/or tricarboxylic acid cycle. Without a doubt, these metabolic pathways are of great interest to gain a quantitative understanding of the cellular capability to provide precursors, reduction equivalents, or energy for cellular functions and anabolic demands. However, the monitoring of metabolism dynamics in anabolic pathways would likewise be interesting because of the potential use of the data to qualify the biochemical pathway reactions for the production of desired pathway products.

So far, no corresponding studies have been published focusing on anabolic routes. This might be surprising because especially pathway products such as amino acids, vitamins, and antibiotics are of outstanding commercial interest and thus at the forefront of metabolic engineering. However, if the focus changes from catabolic to anabolic reactions, two inherent drawbacks must be 
taken into account that significantly hamper the 'stateof-the-art' glucose pulse approach.

(i) Analytical Access. While almost all metabolites of the central metabolism can be measured enzymatically (Bergmeyer, 1985) or, most promisingly, with the aid of LC-MS/MS technology (Buchholz et al., 2001; van Dam, 2002), similar analytical approaches for other pathway intermediates are usually lacking. Although tools such as LC-MS/MS offer great promise for quantifying intracellular metabolite concentrations in general, nevertheless at least purified standards of the intermediates are necessary, which are usually not available commercially. This holds also true for the recently published MIRACLE approach (Mashego et al., 2004). Hence, time-consuming chemical synthesis or the isolation (and purification) of the substances from fermentation supernatant using special "knock-out" mutants is the consequence.

(ii) Signal Dilution. The analysis of metabolite concentration profiles of the central metabolism benefits from the relatively short reaction sequence from the signal input (glucose pulse) to its output (metabolite of interest). However, the signal cascade to intermediates of anabolic pathways is significantly longer, thus more branching-off reactions or feedback regulations must be taken into account that finally "dilute" the metabolism dynamics before they reach the desired target metabolite in the anabolic pathway. Corresponding experimental observations have been published by Schmitz et al. (2002) exemplarily studying the aromatic amino acid pathway. Hence, the analysis of anabolic pathways dynamics not only necessitates a sufficient stimulation of substrate uptake but also a fortification of the carbon flux into the anabolic pathway of interest to ensure an appropriate pathway stimulation.

Despite these potential drawbacks, this contribution aims at stimulating, monitoring, and analyzing pathway dynamics by metabolic profiling (Fiehn, 2001). These data are intended to provide a quantitative understanding of pathway mechanisms, in the sense of "modern" metabolic engineering (Westerhoff, 2001).

As an example, the aromatic amino acid pathway in $E$. coli for the production of L-phenylalanine has been chosen because of the extensive knowledge about this route (Pittard, 1996) combined with its commercial importance (Frost and Lievense, 1993; Schmid et al., 2001; Bongaerts et al., 2001) at the same time embracing the typical drawbacks as mentioned above.

It will be shown that a concerted approach of strain construction, experimental procedure, LC-MS/MS, and data analysis allows to overcome the drawbacks, thus offering the advantage of observing in vivo pool oscillations, which provide valuable insight into underlying reaction mechanisms. The statistical analysis of the metabolism dynamics, which finally introduces a simple, data-driven criterion called "pool efflux capacity" (PEC), should be regarded as a first, simple step of data analysis. Nevertheless, it allows us to identify promising metabolic engineering targets for further strain optimization.

\section{Methods}

Strain and Culture Conditions. Based on E. coli K12 LJ110 (Zeppenfeld et al., 2000), two L-phenylalanine production strains with the synonyms $E$. coli A (coding for the genotype $\Delta(p h e A$ tyrA aroF $) / \mathrm{pJF} 119 \mathrm{EH}$ aroFfbr phe $A^{f b r} a m p$ ) and $E$. coli B (coding for the genotype $\Delta(p h e A$ tyrA aroF $) / \mathrm{pJF} 119 \mathrm{EH}$ aroFfbr $p h e A^{f b r}$ aroB amp) were used for the glucose pulse experiments. As indicated, the strains contained chromosomal deletions of the genes tyrA (chorismatemutase/prephenate dehydrogenase), pheA (chorismatemutase/prephenate dehydratase), and $\operatorname{aroF}$ (L-tyrosine-sensitive DAHP-synthase). Plasmids $\mathrm{A}$ and $\mathrm{B}$ were based on the pJF119EH expression vector (Fürste et al., 1986), containing an ampicilline resistance gene and an IPTG-inducible tac-promotor, together with the genes $a r o F^{f b r}$ (Jossek et al., 2001), encoding the L-tyrosine feedback-resistant DAHP synthase; $p h e A^{f b r}$, coding for the L-phenylalanine feedback-resistant chorismatemutase/prephenate dehydratase); and $\operatorname{aro} B$, coding for the 3-DHQ synthase (only in B). Hence, both strains were L-tyrosine-auxotrophic. Details of $E$. coli A are presented in Gerigk et al. (2002). E. coli B was constructed by Degner and Sprenger (unpublished results).

Cryocultures were stored in Luria-Bertani (LB) medium containing $50 \%$ glycerol at $-80{ }^{\circ} \mathrm{C}$. The precultivation followed the procedure of Gerigk et al. (2002). For fermentations, the following medium has been applied: $3.0 \mathrm{~g} / \mathrm{L} \mathrm{MgSO}_{4} \cdot 7 \mathrm{H}_{2} \mathrm{O}, 0.015 \mathrm{~g} / \mathrm{L} \quad \mathrm{CaCl}_{2} \cdot \mathrm{H}_{2} \mathrm{O}, 3.0 \mathrm{~g} / \mathrm{L}$ $\mathrm{KH}_{2} \mathrm{PO}_{4}, 1.0 \mathrm{~g} / \mathrm{L} \mathrm{NaCl}, 5.0 \mathrm{~g} / \mathrm{L}\left(\mathrm{NH}_{4}\right)_{2} \mathrm{SO}_{4}, 0.075 / 0.1 \mathrm{~g} / \mathrm{L}$ $\mathrm{FeSO}_{4} \cdot 7 \mathrm{H}_{2} \mathrm{O} /$ sodium citrate, $0.075 \mathrm{~g} / \mathrm{L}$ thiamine, $0.3 \mathrm{~g} / \mathrm{L}$ L-Tyr, $0.1 \mathrm{~g} / \mathrm{L}$ ampicillin, $15 \mathrm{~g} / \mathrm{L}$ glucose, and $1.5 \mathrm{~mL} / \mathrm{L}$ trace element solution containing $2.0 \mathrm{~g} / \mathrm{L} \mathrm{Al}_{2}\left(\mathrm{SO}_{4}\right)_{3}$. $18 \mathrm{H}_{2} \mathrm{O}, 0.75 \mathrm{~g} / \mathrm{L} \mathrm{CoSO}_{4} \cdot 7 \mathrm{H}_{2} \mathrm{O}, 2.5 \mathrm{~g} / \mathrm{L} \mathrm{CuSO}_{4} \cdot 5 \mathrm{H}_{2} \mathrm{O}, 0.5$ $\mathrm{g} / \mathrm{L} \mathrm{H}_{3} \mathrm{BO}_{3}, 24 \mathrm{~g} / \mathrm{L} \mathrm{MnSO} \cdot \mathrm{H}_{2} \mathrm{O}, 3.0 \mathrm{~g} / \mathrm{L} \mathrm{Na}_{2} \mathrm{MoO}_{4} \cdot 2 \mathrm{H}_{2} \mathrm{O}$, $2.5 \mathrm{~g} / \mathrm{L} \mathrm{NiSO}_{4} \cdot 6 \mathrm{H}_{2} \mathrm{O}$, and $15.0 \mathrm{~g} / \mathrm{L} \mathrm{ZnSO}_{4} \cdot 7 \mathrm{H}_{2} \mathrm{O}$. The same medium was used for precultures with the following changes: $0.3 \mathrm{~g} / \mathrm{L} \mathrm{MgSO}_{4} \cdot 7 \mathrm{H}_{2} \mathrm{O}, 0.1 \mathrm{~g} / \mathrm{L} \mathrm{NaCl}, 0.0075 \mathrm{~g} / \mathrm{L}$ thiamine $\cdot \mathrm{HCl}, 0.08 \mathrm{~g} / \mathrm{L}$ L-Tyr, $5.0 \mathrm{~g} / \mathrm{L}$ glucose, and, additionally, $12 \mathrm{~g} / \mathrm{L} \mathrm{K}_{2} \mathrm{HPO}_{4}$ (final $\mathrm{pH}$ 7.2).

Fed-batch fermentations ( $7 \mathrm{~L}$ ) were conducted in a 20 L bioreactor (ATGU503, Infors AG, CH) at $37^{\circ} \mathrm{C}, \mathrm{pH} 6.5$ (titration with $\mathrm{NH}_{4} \mathrm{OH}$ ), controlling dissolved oxygen (DO) via an amperometric electrode (Mettler-Toledo, Germany) and measuring oxygen and carbon dioxide content in the exhaust gas. LabView was used for data acquisition. The L-tyrosine feed was realized with a Dosimat 665 (Metrohm AG, Switzerland), ensuring excess L-tyrosine $(0.20-0.30 \mathrm{~g} / \mathrm{L})$ during the whole fermentation. The description of the sampling procedure and the analytical methods for glucose, L-tyrosine, and L-phenylalanine determination are given in Gerigk et al. (2002b). The supply of the sole carbon source, glucose, was used to control cell growth. This resulted in a nonlimited batch and fed-batch phase (permanent glucose level $3-5 \mathrm{~g} / \mathrm{L}$ ) and a growth-limited glucose supply (50 g/h) after an optical density $\left(\mathrm{OD}_{650}\right)$ of 50 was achieved. The glucoselimited, tyrosine-rich period lasted for $20 \mathrm{~min}$ (strain A) and $45 \mathrm{~min}$ (strain B) before the glucose pulse $(50 \mathrm{~mL}$, $420 \mathrm{~g} / \mathrm{L}$ ) was added automatically to the bioreactor, thus shifting the concentration up to $3 \mathrm{~g} / \mathrm{L}$, while rapid sampling was started at the same time.

Rapid Sampling Procedure. Using the sampling device described elsewhere (Schäfer et al., 1999), a sampling frequency of $4-5 \mathrm{~s}^{-1}$ of $5 \mathrm{~mL}$ was achieved. Sampling tubes contained $15 \mathrm{~mL}$ of a $70 \mathrm{mM}$ HEPES $60 \%$ $\mathrm{v} / \mathrm{v} \mathrm{MeOH}$ solution at $-50{ }^{\circ} \mathrm{C}$ to rapidly stop cellular metabolism. After centrifugation at $-20{ }^{\circ} \mathrm{C}$, cells were resuspended in $1 \mathrm{~mL}$ of $-20^{\circ} \mathrm{C} 50 \% \mathrm{v} / \mathrm{v} \mathrm{MeOH}$, extracted with $4 \mathrm{~mL}$ of $-20{ }^{\circ} \mathrm{C} 43.75 \%$ perchloric acid, and neutralized with saturated $\mathrm{K}_{2} \mathrm{CO}_{3}$ solution after one freeze-thaw cycle. After centrifugation, cell extract samples were analyzed with LC-MS. Technical problems of the experimental setup reduced the prepulse observation window from -4.5 to $-2.5 \mathrm{~s}$ for strain $\mathrm{B}$ and reduced the postpulse observation window of strain A to $18 \mathrm{~s}$.

Analytical Access. Cell extract samples were analyzed with a newly developed LC-MS/MS method. The 
Table 1. Overview of Enzyme Kinetic Qualities Found by in Vitro Enzyme Studies Using $E$. coli

\begin{tabular}{|c|c|c|c|}
\hline reaction & $\begin{array}{l}\text { sub- } \\
\text { strate }\end{array}$ & $\begin{array}{c}K_{\mathrm{m}}(\mu \mathrm{M}) \\
\quad \text { or } \\
s^{0.5}(\mu \mathrm{M})^{a}\end{array}$ & ref \\
\hline \multicolumn{4}{|l|}{ DAHP synthase } \\
\hline \multirow{2}{*}{$\begin{array}{l}\text { AroF } \\
\text { (Tyr-sensitive) }\end{array}$} & $\mathrm{E} 4 \mathrm{P}$ & $80-90$ & Ramilo and Evans (1997) \\
\hline & PEP & $5.8-13$ & Schoner and Herrmann (1976) \\
\hline \multirow{2}{*}{$\begin{array}{l}\text { AroG } \\
\text { (Phe-sensitive) }\end{array}$} & & $\sim 80 ; 900$ & Simmonds (1950) \\
\hline & PEP & $\sim 5 ; 80$ & Simpson and Davidson (1976) \\
\hline \multirow{2}{*}{$\begin{array}{l}\text { AroH } \\
\text { (Trp-sensitive) }\end{array}$} & $\mathrm{E} 4 \mathrm{P}$ & $35^{*}$ & Mc Candliss et al. (1978) \\
\hline & PEP & $5.3^{*}$ & Akowski and Bauerle (1997) \\
\hline \multirow{2}{*}{$\begin{array}{l}\text { DHQ synthase } \\
\text { (EC 4.2.3.4) } \\
\text { AroB }\end{array}$} & DAHP & $4 ; 30-50$ & Mehdi et al. (1987) \\
\hline & & & $\begin{array}{l}\text { Le Marchal et al. (1980) } \\
\text { Maitra and Sprinson (1978) } \\
\text { Widlanski et al. (1989) }\end{array}$ \\
\hline \multirow{2}{*}{$\begin{array}{l}\text { DHQ dehydratase } \\
\text { (EC 4.2.1.10) } \\
\text { AroD }\end{array}$} & 3-DHQ & $10-18$ & Kleanthous et al. (1992) \\
\hline & & & $\begin{array}{l}\text { Chaudhuri et al. (1986) } \\
\text { Chaudhuri et al. (1987) }\end{array}$ \\
\hline \multirow[t]{2}{*}{$\begin{array}{l}\text { shikimate } \\
\text { dehydrogenase } \\
\text { AroE } \\
\text { shikimate kinase } \\
\text { (EC 2.7.1.71) } \\
\text { AroK (SK I) }\end{array}$} & 3-DHS & not found & \\
\hline & $\begin{array}{l}\text { SHI } \\
\text { ATP }\end{array}$ & $\begin{array}{l}\sim 5000 \\
\sim 160\end{array}$ & $\begin{array}{l}\text { de Feyter (1987) } \\
\text { de Feyter (1986) }\end{array}$ \\
\hline AroL (SKII) & SHI & 200 & \\
\hline \multirow{2}{*}{$\begin{array}{l}\text { EPSP synthase } \\
\text { (EC 2.5.1.19) } \\
\text { AroA }\end{array}$} & $\begin{array}{l}\text { ATP } \\
\text { S3P }\end{array}$ & $\begin{array}{l}160 \\
2.5-3.6\end{array}$ & Duncan et al. (1984) \\
\hline & PEP & $10-20$ & $\begin{array}{l}\text { Gruys et al. (1992) } \\
\text { Lewendon and Coggins (1987) }\end{array}$ \\
\hline
\end{tabular}

${ }^{a} K_{\mathrm{m}}$ values for Michelis-Menten-type reactions as well as $s_{0.5}$ values for sigmoidal kinetics are given. Values for sigmoidal kinetics are indicated by an asterisk. In case of AroE, no corresponding information was found in the literature.

hardware consisted of an Agilent1100 HPLC, a HTC Pal autosampler (CTC Analytics), and a TSQ Quantum triple quadrupole mass spectrometer (ThermoFinnigan). The LC method uses the Nucleodex beta-OH phase described in Buchholz et al. (2001), and LC flow is split to direct only $100 \mu \mathrm{L} / \mathrm{min}$ to the TSQ Quantum mass spectrometer, which was operated in multiple reaction monitoring (MRM) mode with an ESI source in negative ionization mode and centroid data acquisition. Nitrogen was used as a sheath (50) and auxiliary (15) gas, Q2 collision gas (argon) was set to 1.5 mTorr, capillary voltage to $4 \mathrm{kV}$, capillary temperature to $375{ }^{\circ} \mathrm{C}$, scan time to $150 \mathrm{~ms}$, and Q1 + Q3 peak width to 0.7 atomic mass unit (amu). The compound specific MS/MS parameters, meaning the parent and product ion and the optimized collision energy for the MS/MS transition of each metabolite, are given in Table 2. These parameters were determined using standard solutions of the metabolites. Without a standard solution it is not possible to set up the MS detector in the highly sensitive and quantitative MRM mode for the measurement. To correct for matrix effects in the LCMS/MS analysis, quantification was done by the standard addition method (Bader, 1980).

Preparation of Metabolites from Aromatic Biosynthesis. Except for shikimate, no metabolite from aromatic biosynthesis measured in this study is commercially available. Consequently, these compounds had to be produced and purified before starting the analytical method development. Because of their high functionality, the metabolites are difficult to prepare using classical chemical approaches. By applying genetic engineering techniques, a smarter access can be realized by using specially constructed, blocked $E$. coli strains that release
Table 2. Compound Specific MS/MS Parameters Showing Parent Ion, Optimal Product Ion, and Optimized Collision Energy for the MS/MS Transition

\begin{tabular}{lccc}
\hline \multicolumn{1}{c}{ compound } & $\begin{array}{c}\text { parent } \\
\text { ion } \\
(\mathrm{m} / \mathrm{z})\end{array}$ & $\begin{array}{c}\text { product } \\
\text { ion } \\
(\mathrm{m} / \mathrm{z})\end{array}$ & $\begin{array}{c}\text { collision } \\
\text { energy } \\
(\mathrm{eV})\end{array}$ \\
\hline $\begin{array}{l}\text { 3-deoxy-D-arabino-heptulosonate } \\
\quad \text { 7-phosphate (DAHP) }\end{array}$ & 287.1 & 79.0 & 45 \\
3-deoxy-arabino-heptulosonate (DAH) & 207.2 & 87.0 & 12 \\
3-dehydroquinate (3-DHQ) & 189.1 & 170.8 & 12 \\
3-dehydroshikimate (3-DHS) & 171.1 & 127.0 & 12 \\
shikimate (SHI) & 173.1 & 93.0 & 17 \\
shikimate 3-phosphate (S3P) & 253.1 & 97.0 & 17 \\
phosphoenolpyruvate (PEP) & 167.0 & 79.0 & 13 \\
pentose 5-phosphates (P5P) & 229.1 & 97.0 & 15
\end{tabular}

the desired metabolite(s) into the supernatant using glucose as a readily available starting material. From the culture supernatant the compounds can be purified using preparative ion chromatography or extraction steps. The metabolites DAH, DAHP, DHS, and S3P were isolated using this concept. Fermentation was performed in a 2 $\mathrm{L}$ Labfors bioreactor (Infors) in fed-batch mode with glucose feeding, medium composition, and fermentation handling as described by Schmitz et al. (2002). DAH was produced from an $a r o B$-negative $E$. coli mutant that accumulated $160 \mathrm{mM}$ DAH within $30 \mathrm{~h}$ in a fed-batch fermentation. It was isolated from culture supernatant using cation and anion chromatography (Mehdi et al., 1987). The isolation of a DAHP standard from the same supernatant using the described procedure yielded only a small amount of partially purified qualitative standard that was, nevertheless, pure enough to set up the LCMS/MS method parameters for DAHP. 3-DHS was isolated from the culture supernatant of an $a r o E$-negative $E$. coli mutant that produced $120 \mathrm{mM}(30 \mathrm{~h})$ in a fedbatch fermentation. Purification was performed by applying liquid-liquid extraction (Dell and Frost, 1993). On the basis of an aroA-negative $E$. coli mutant, $27 \mathrm{mM}$ S3P $(30 \mathrm{~h})$ accumulated and was isolated with ion chromatography from culture supernatant (Coggins et al., 1987). All knock-out mutants are described in Schmitz et al. (2002). 3-DHQ was chemically synthesized from quinate using nitric acid oxidation following the procedure of Grewe and Jeschke (1956) and was purified from the reaction mixture with ion chromatography.

Data Analysis. Measured data as shown in Figure 3 were smoothed using fast Fourier transformation (FFT) as implemented in Origin (OriginLab Corp.). A smoothing window of five interpolation points was chosen after preliminary statistical analysis, because higher (seven points) as well as lower (three points) smoothing degree gave very similar statistical and PEC (see next paragraph) results. For statistical correlation studies, the FFT-smoothed pool data were used as input variables for applying eq 1, thus calculating the "observations" (i.e. standardized pool velocities, see eq 1 ) of each variable (pool) for each sample. As a result, one matrix of each pulse experiment was formulated, which was further studied with the aid of the standard correlation analysis function (corrcoeff) implemented in MATLAB (The Mathworks Inc.).

Further data analysis was performed with the aid of a novel criterion, called pool efflux capacity (PEC). This criterion is purely based on concentration data and aims at a simple, data-driven analysis of dynamic metabolite courses. It was motivated by the idea to allow a fast, primary data analysis without making it necessary to identify complex metabolism models consisting of, for instance, mechanistic approaches. 
This is why the standardized pool velocity $v(1 / \mathrm{s})$ was introduced, taking into account the prepulse concentration $\left(\bar{c}_{i}\right)$ and the current concentration of pool $i$ as follows

$$
v_{i}=\frac{1}{\bar{c}_{i}} \frac{\mathrm{d} c_{i}(t)}{\mathrm{d} t}
$$

The standardized pool velocity thus resembles a little the "left side" of an ordinary mass balance definition with respect to the pool species $i$. The value mirrors the resulting net rate of all influx and effluxes of this pool. High positive values thus indicate a fast pool size increase (i.e. the influx is higher than the efflux), while high negative values represent a rapid decrease, both related to the prepulse concentration. Using this criterion, concentration courses were transferred to velocity series. The amplitudes of these series are ranked with respect to the minimum standardized pool velocity $\left[\min \left(v_{i}\right)\right]$, i.e., the highest (negative) standardized pool velocity of each species was selected, which resembles the maximum net efflux rate of a pool.

The guiding idea was to define a simple criterion called "pool efflux capacity" (PEC) that aims at mirroring the distributed flux control within a reaction sequence [according to the metabolic control analysis (MCA) theory] by comparing the maximum net efflux rates of each step, thus relating the enzyme with the strongest flux control to the slowest pool decline.

\section{Results and Discussion}

Fermentation Strategy for Pulse Experiments. At the beginning of the study, preliminary experiments have been performed to identify an appropriate glucose pulse scenario such that the experimental conditions are-more or less - comparable to those used for microbial L-phenylalanine synthesis. Consequently, the results of the pulse experiments should be readily transferable to production conditions. Therefore, it was decided to study metabolism dynamics with nongrowing, L-Phe producing cells (see Gerigk et al., 2002a,b; Rueffer et al., 2004).

Figure 1 depicts the resulting experimental procedure that has been applied for the two L-Phe producers $E$. coli $\mathrm{A}$ and $\mathrm{B}$. At the beginning, glucose and tyrosine were consumed until a dual feed of glucose and tyrosine was installed such that ample tyrosine $(0.2-0.3 \mathrm{~g} / \mathrm{L})$ and glucose $(3-5 \mathrm{~g} / \mathrm{L})$ levels have been maintained during the subsequent cell growth phase. After about $9-10 \mathrm{~h}$ process time, cell growth was halted, because the glucose feed has been reduced drastically, thus causing glucoselimiting conditions, while the tyrosine concentration was still in excess. After about 30-45 min, the glucose pulse was added such that an extracellular glucose level of 3 $\mathrm{g} / \mathrm{L}$ was installed (data not shown), thus ensuring a saturated glucose uptake for the cells. It is noteworthy that details about the glucose pulse, the mixing time, and the rapid sampling device are given elsewhere (Schäfer et al., 1999, Oldiges and Takors, 2004). Immediately before and after the glucose pulse, rapid cell sampling was performed to monitor the intracellular metabolism dynamics, as described in the preceding sections.

Basics of Glucose Pulse Analysis. Accordingly, cells were stimulated by the sudden rise of glucose uptake, changing the intracellular levels of glucose 6-phosphate (G6P), phosphoenolpyruvate (PEP), and pyruvate (PYR) immediately, because of the underlying phosphoenolpyruvate:carbohydrate phosphotransferase system (pts) in $E$. coli. As a consequence, a negative signal response for PEP (expressed by dropping PEP concentrations) originating

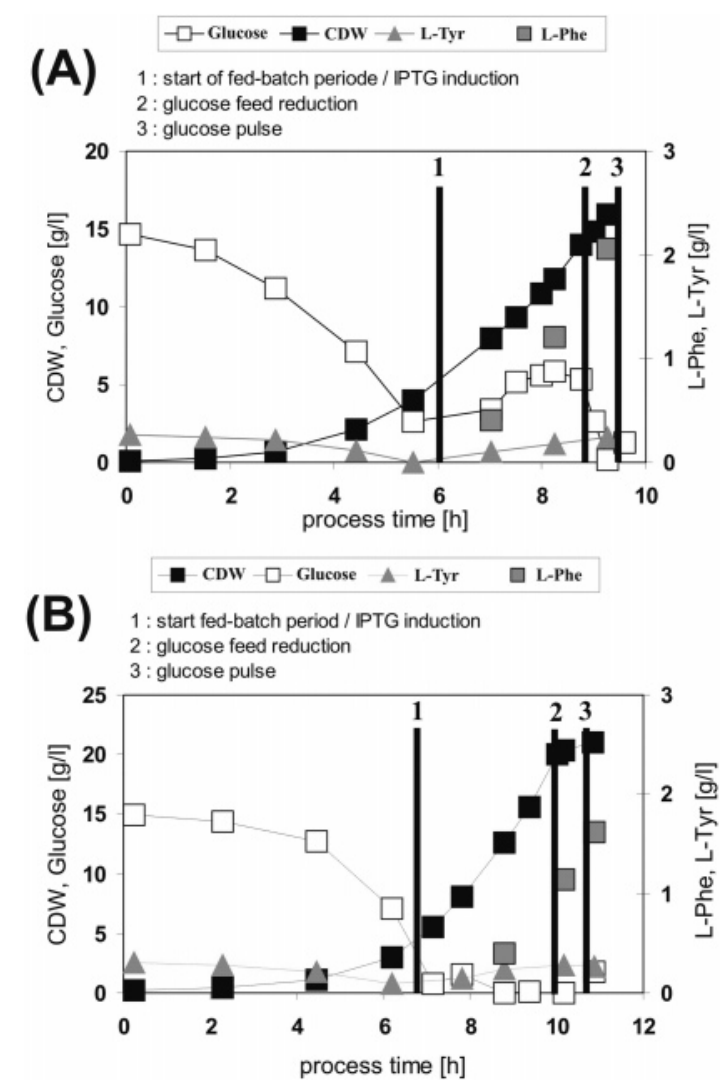

Figure 1. Fermentation courses of the glucose pulse experiments (A and B) using the tyrosine-auxotrophic L-phenylalanine (L-Phe) producers $E$. coli strains A and B, respectively (for definitions of strain synonyms, see the text). After glucose has been depleted during the primary batch phase, a glucose feed has been started such that a permanent level of $3-5 \mathrm{~g} / \mathrm{L}$ substrate was installed. After $9-10 \mathrm{~h}$ process time, this feed was reduced drastically, concomitantly ensuring sufficient tyrosine supply, before the glucose pulse was added after $30-45$ min of glucose limitation.

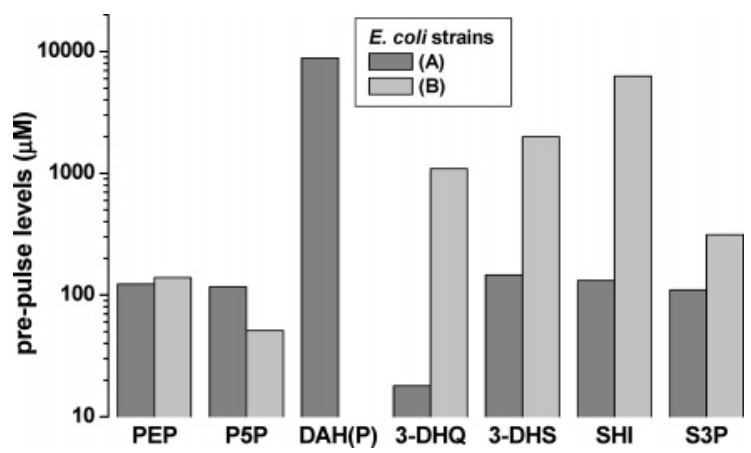

Figure 2. Comparison of different intracellular mean metabolite levels analyzed during the last $2.5-5 \mathrm{~s}$ before the glucose pulse. Two different genotypes were used as L-phenylalanine producers. Strain A only possessed an $a r o F^{f b r}$ overexpression, while strain B contained $a r o F^{f b r}$ and $a r o B$ overexpression. The precursors phosphoenolpyruvate (PEP), the lumped pentose phosphate pool (P5P), DAH(P), 3-dehydroquinate (3-DHQ), 3-dehydroshikimate (3-DHS), shikimate (SHI), and shikimate 3-phosphate (S3P) are shown.

from the positive glucose stimulation can be expected. Within this study, we decided to focus our interest on the linear, upstream part of the aromatic amino acid pathway, namely the conversion of the precursors PEP and erythrose 4-phosphate (E4P) via five subsequent reactions to shikimate 3 -phosphate (S3P); see Figure 3. This was motivated by the fact that the subsequent reaction from S3P to 5-enolpyruvoylshikimate 3-phos- 

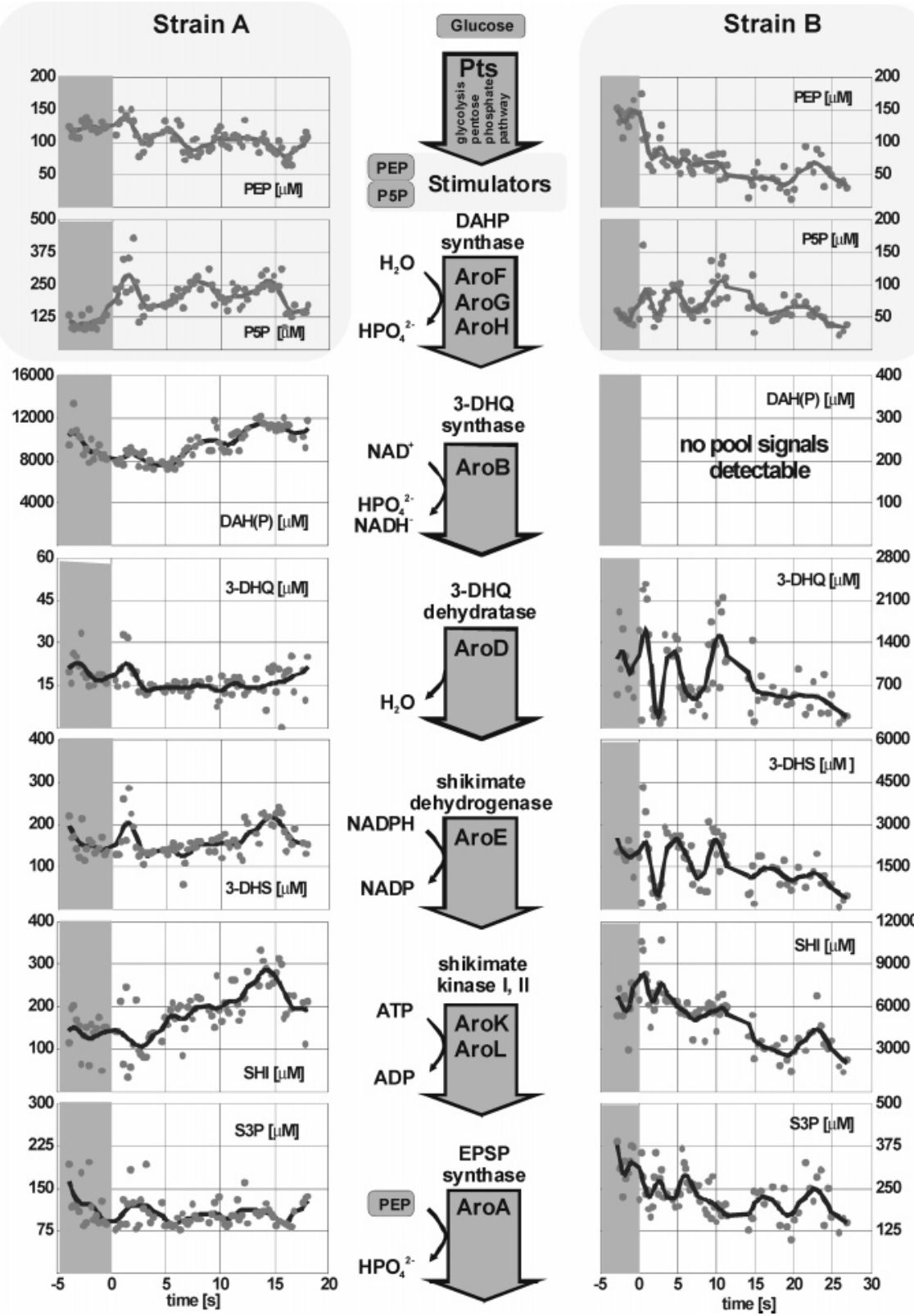

Figure 3. Metabolism dynamics in the aromatic amino acid pathway, forced by the independent stimulators phosphoenolpyruvate (PEP) and the lumped pentose phosphate pool (P5P) immediately after a glucose pulse. Left, strain A (aroF ${ }^{f b r}$ overexpression). Right, strain B (overexpression of $a r o F^{f b r}$ and $a r o B$ ). PEP and erythrose 4-phosphate (E4P), the latter represented by P5P, are metabolized via the three DAHP synthase isoenzymes AroF, AroG, and AroH into DAH(P). 3-Dehydroquinate (3-DHQ) is synthesized from DAHP using 3-DHQ synthase. $\mathrm{H}_{2} \mathrm{O}$ is separated in the subsequent dehydratase catalyzed reaction leading to 3-dehydroshikimate, which is further reduced to shikimate via shikimate dehydrogenase. Shikimate kinases I and/or II activate this substrate to shikimate 3-phosphate, the substrate for EPSP production together with additional PEP, which is no longer followed by analysis. The line graphs represent the FFT smoothed raw data.

phate (EPSP) again needs PEP for condensation, thus leading to an "unusual" second stimulation of the pathway. Additionally, the following, commercially available metabolites, chorismate and prephenate, are chemically unstable, which would significantly hamper their use for metabolic profiling analysis.

As indicated in the Methods, metabolites such as 3-deoxy-D-arabino-heptulosonate 7-phosphate (DAHP), its dephosphorylated derivative (DAH), 3-dehydroquinate, 3-dehydroshikimate, and shikimate 3-phosphate were not commercially available and had to be isolated and purified from cell suspension after their production using $E$. coli knock-out mutants. In all experiments, E4P was not detectable, which is in agreement with the previous observations of Williams et al. (1980). They critically reviewed in vivo measured $\mathrm{E} 4 \mathrm{P}$ levels, concluding that only very low E4P pools should be expected in vivo (if at all), because of E4P's tendency to react into dimeric forms. Also, Ruijter and Visser (1999) outlined that no E4P could be detected in Aspergillus niger strains, although the respective detection limit was about 25 pmol. Considering that even $\mathrm{AroF}^{\mathrm{fbr}}$-fortified strains were used in this study, no significant E4P pools should be expected. Therefore, the lumped pentose phosphate pool P5P was used as a reference instead, allowing at least some speculations about the E4P supply via the transketolase (tktA) reaction. In the case of DAHP, the small amount of isolated standard showed some impurities, making it difficult to apply it for quantification and thus motivating the authors to use the dephosphorylated derivative DAH instead. This decision was justified by the experimental finding that the ratio of $\mathrm{DAH}$ concen- 
trations versus DAHP peak area was constant at $3.3 \pm$ $0.308 \mathrm{nM} /$ peak area in all LC-MS/MS measurements. Thus, a common $\mathrm{DAH}(\mathrm{P})$ pool is considered in the following discussion. Metabolite courses reflecting the dynamics in central metabolism were also measured. But, for the sake of brevity, their presentation is reduced to the essentials, namely the aromatic amino acid pathway precursors PEP and P5P.

Pulse Experiments with Strain A. As shown in Figure 2 (dark grey columns), the mean intracellular prepulse pools found in $E$. coli strain A were most at physiological levels of several $100 \mu \mathrm{M}$, apart from DAH(P), which showed a very large pool of about $10 \mathrm{mM}$. From this we concluded that the huge $\mathrm{DAH}(\mathrm{P})$ pool was a consequence of the single overexpression of $a r o F^{f b r}$ caused by a high flux into this pool, which could not be compensated by a corresponding downstream reaction. The considerable inequality of pool sizes between DAH(P) and the subsequent $3-\mathrm{DHQ}$ of almost 3 orders of magnitude should be noted.

Figure 3 (left column) presents the results of the glucose pulse experiment using strain A. Before the pulse, the PEP and the P5P pool were in pseudo-steadystate, which is indicated by rather constant pool sizes (PEP, $121 \pm 9 \mu \mathrm{M}$; P5P, $107 \pm 25 \mu \mathrm{M}$ ) compared to the following dynamics. After the pulse, both pools changed significantly, as was indicated by a strong increase followed by the oscillating behavior of P5P and PEP. Both pools served as precursors for the DAHP-synthase. Table 1 indicates that none of the three DAHP-synthase isoenzymes is supposed to be limited by PEP supply, because the measured intracellular levels of $70-140 \mu \mathrm{M}$ were well above the reported $K_{\mathrm{m}}$ values. In the case of $\mathrm{E} 4 \mathrm{P}$, no pools were measured, thus indicating an almost entire limitation of DAHP synthase activity $\left(K_{\mathrm{M}, \mathrm{E} 4 \mathrm{P}} \sim 35-900 \mu \mathrm{M}\right)$ at first glance. However, it should be queried whether dissolved E4P really exists in cell cytoplasm because of its high affinity for dimerization, thus supporting the hypothesis of E4P channeling via DAHP-synthase (Williams et al., 1980). Hence, we concluded that pulse response into the aromatic amino acid pathway in strain A was not limited by PEP or E4P, but it was buffered by the subsequent, huge $\mathrm{DAH}(\mathrm{P})$ pool. Most presumably, the buffering occurred as a consequence of the significant AroB inhibition in the presence of 8 to $12 \mathrm{mM} \mathrm{DAH}$, taking into account the previous findings of Maitra and Sprinson (1978), who observed that already $5 \mathrm{mM}$ DAH caused $72 \%$ AroB inhibition. As a consequence, glucose pulse signaling to the subsequent pools was hampered.

The pure analysis of the concentration courses can only provide a limited insight into the underlying reaction mechanisms. Preferably, the time-variant concentration courses should be used for a model-based system analysis, which is currently done but not yet finished. However, as a primary step, the standardized pool velocitites $v(1 / \mathrm{s})$ have been calculated (see Data Analysis), as indicated in Figure 4. It is shown that the precursor P5P obviously represented the most perturbed pool, which was still oscillating at the end of the observations after $18 \mathrm{~s}$. This held also true for shikimate. However, no common behavior such as in-phase oscillations can be observed in the pools, neither before nor after the pulse. The most striking effect is the inertia of the $\mathrm{DAH}(\mathrm{P})$ pool, which is also detected by pool velocity analysis, showing almost unchanged values about zero.

Motivated by this finding, we ranked the observed amplitudes with respect to the minimum standardized pool velocity $\left[\min \left(v_{i}\right)\right]$, i.e., the highest (negative) standardized pool velocity of each species was selected that
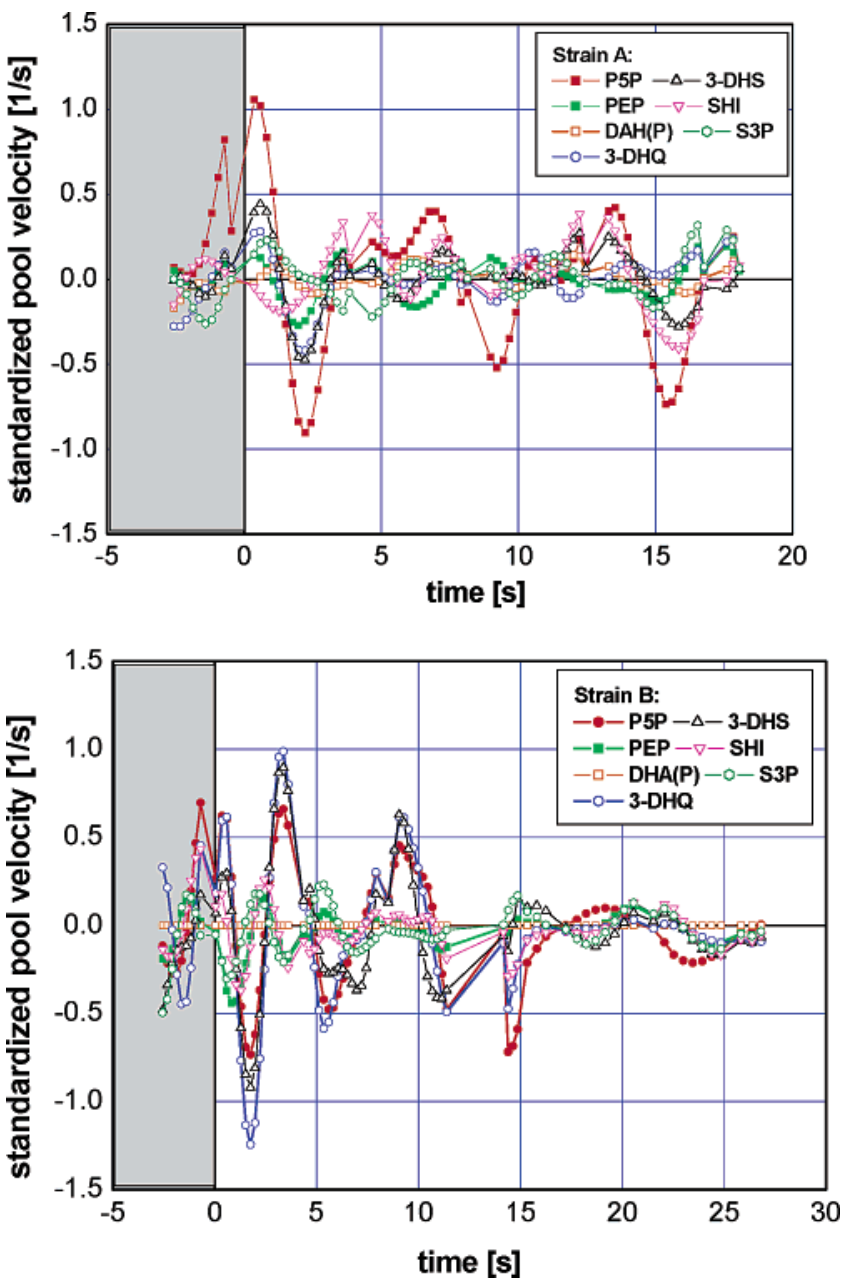

Figure 4. Courses of the standardized pool velocities before and after the glucose pulse using strains A and B. The following symbols were used: P5P, full, red circle; PEP, full, green rectangle; $\mathrm{DAH}(\mathrm{P})$, open, orange rectangle; 3-DHQ, open, blue circle; 3-DHS, open, up black triangle; SHI, open, down magenta triangle; S3P, open, green hexagon.

resembles the maximum net efflux rate of a pool, and thus deriving the "pool efflux capacities" (PEC) (see Data Analysis).

Following the procedure, pool efflux capacities (1/s) of $0.09,0.42,0.48,0.41$, and 0.22 were calculated for $\mathrm{DAH}(\mathrm{P}), 3-\mathrm{DHQ}$, 3-DHS, SHI, and S3P (see Figure 5). According to the working hypothesis that the lowest pool efflux capacity [here $\mathrm{DAH}(\mathrm{P})]$ indicates an enzyme exerting high flux control, the 3-dehydroquinate synthase, encoded by $\operatorname{aro} B$, was identified as a promising metabolic engineering target, which is in agreement with previous studies (Snell et al., 1996; Pittard, 1996; Yi et al., 2002). Hence, strain B was constructed.

It is noteworthy that the PEC criterion cannot readily be compared with the classical definitions given by the metabolic control analysis (MCA). In this contribution, highly dynamic data were analyzed while the classical MCA typically uses small deviations of steady-state fluxes to identify flux control coefficients, elasticity coefficients, etc. Beyond it, MCA approaches for the analysis of highly dynamic data have been published almost recently (Visser and Heijnen, 2003). MCA has rejected the idea of a single rate-limiting step (bottleneck) in a (linear) pathway and has introduced the shared flux control by all enzymatic reactions instead (Westerhoff and Kell, 1996). Likewise, the different PEC values (Figure 5) should be interpreted, although the math- 


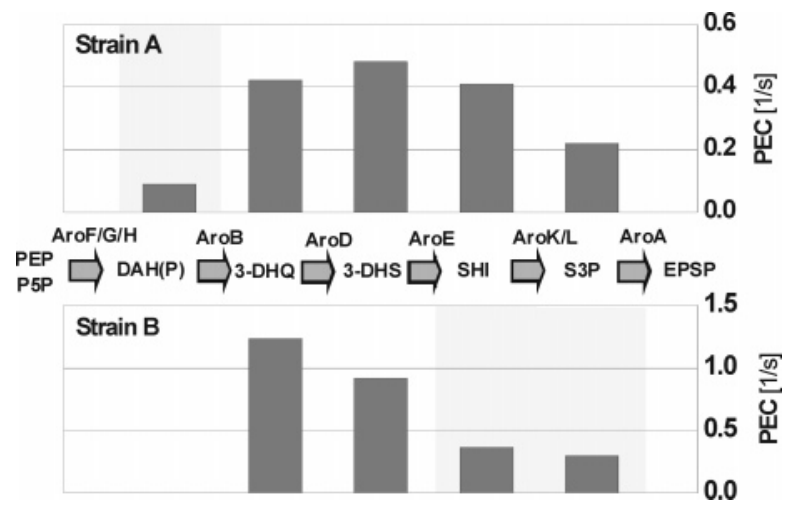

Figure 5. Pool efflux capacity (PEC) criterion of strains A and $\mathrm{B}$ identified after glucose pulse experiments with respect to the pools DAH(P), 3-DHQ, 3-DHS, SHI, and S3P. Furthermore, the independent stimulators PEP and P5P as well as EPSP, the subsequent pool of the reaction sequence, are given. Additionally, the enzymes involved with their abbreviated names are shown. The shaded background indicates the pool whose subsequent reaction exerts relatively high flux control (compared to the other reactions) in this linear pathway.

ematical relation between PEC values and flux control coefficients has not yet been studied and identified.

Pulse Experiments with Strain B. Figure 2 (light grey columns) gives an overview of the mean prepulse concentrations in the $E$. coli strain B possessing an overexpression of $a r o F^{f b r}$ and $a r o B$. Most strikingly, no intracellular $\mathrm{DAH}(\mathrm{P})$ could be measured, although the intracel-

lular levels of PEP and P5P remained almost constant compared to the results of strain A. This allows the conclusion that the previously existing AroB limitation has been alleviated. However, the finding coincided with a strong increase of the pool sizes downstream of $\mathrm{DAH}(\mathrm{P})$ in strain B, indicating additional flux limitations further down.

Significant differences were also observed following the intracellular metabolite dynamics (Figure 3 ). The precursors PEP and P5P showed different pool courses in strain $\mathrm{B}$, which held especially true for the decreasing PEP pool. Very strong oscillations were found in the pools of 3-DHQ and 3-DHS, but also in SHI and S3P. The high DAHP affinity of $\mathrm{AroB}$, the increased AroB concentration, and the missing DAH inhibitor were most presumably the reasons for the fast DAHP/3-DHQ conversion without an intermediary accumulation of $\mathrm{DAH}(\mathrm{P})$. It is noteworthy that the initial jump of the 3-DHQ and 3-DHS pool size is not fully covered by the FFT-smoothing approach.

Again, the standardized pool velocities were calculated and presented in Figure 4. In comparison to the results of strain A, pool velocities of strain B show significant differences expressed by damped, in-phase fluctuations of the intermediates. Owing to the removed $\mathrm{DAH}(\mathrm{P})$ buffer in strain $\mathrm{B}$, precursor stimulations are now signaled down via DAHP, leading to in-phase oscillations of the (subsequent) pool velocities of 3-DHQ and 3-DHS during the observation period of $27 \mathrm{~s}$. Both oscillations appear to be in-phase with P5P. With respect to S3P, a strong correlation with the PEP pool velocity can be assumed, which originates most presumably from stoichiometric conversion of S3P and PEP into EPSP via EPSP-synthase together with the refilling reactions via $\mathrm{DAH}(\mathrm{P})$.

Correlation Analysis. On the basis of the standardized pool velocities shown in Figure 4 , a correlation analysis was performed taking into account all postpulse data observed with strain B (see Figure 6). Most strik-

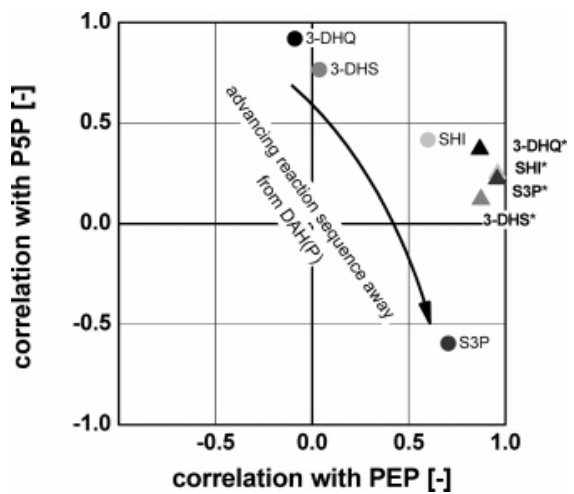

Figure 6. Correlation analysis of the aromatic amino acid pathway intermediates 3-DHQ, 3-DHS, SHI, and S3P with the stimulating precursors $\mathrm{P} 5 \mathrm{P}$ and PEP in the pulse experiment with strain $\mathrm{B}$. DAH(P) is not considered because no significant metabolites could be measured. Circles indicate the correlation results based on all postpulse pool velocity data. Triangles labeled with an asterisk are calculated by correlation analysis based on all data later than $17.5 \mathrm{~s}$.

ingly, the correlation analysis reveals declining reliance on the precursor $\mathrm{P} 5 \mathrm{P}$ with advancing reaction sequence. In analogy, the dependence on PEP grew from 3-DHQ via 3-DHS and SHI to S3P. While P5P was correlated with 3-DHQ and 3-DHS with 0.92 and 0.77 , respectively, the inverse was found for S3P, which was negatively correlated $(-0.6)$ with $\mathrm{P} 5 \mathrm{P}$ and positively correlated (0.71) with PEP

As indicated in Figure 4, a high positive correlation (0.91) was found between 3-DHQ and 3-DHS, allowing the conclusion that the AroD-catalyzed $\mathrm{H}_{2} \mathrm{O}$ separation was very fast. This corresponds to the in vitro measured, low $K_{\mathrm{m}}$ value for 3 -DHQ $(10-18 \mu \mathrm{M}$; see Table 1$)$, which was far below the intracellular substrate level. Hence, a constant 3-DHS production rate must have occurred, giving rise to the conclusion that reaction rates of the subsequent shikimate dehydrogenase (AroE) must have been variable to cause 3 -DHS pool oscillations as observed. As a consequence, high $K_{\mathrm{m}}$ values for 3-DHS or NADPH of AroE could be assumed.

Significantly changing shikimate kinase reaction rates can also be deduced from the data. In the case of strain $\mathrm{B}$, extraordinary high shikimate levels of several millimolar were found, which gave rise to the assumption that not only AroL but also the isoenzyme AroK was active (see Table 1). The latter is characterized by a very high $K_{\mathrm{m}}$ value that significantly limits AroK activity in wildtype strains (Pittard, 1996) and also in $E$. coli strain A. However, in strain B the total shikimate conversion reflects a superimposition of both enzyme activities, meaning a saturated (constant) AroL activity together with a strongly varying AroK-catalyzed conversion (see Table 1).

The correlation analysis supports the qualitative impression derived from Figure 4 that the intermediates 3-DHS and 3-DHQ were strongly correlated with the precursor P5P while S3P (and also SHI) showed higher correlations with the PEP supply. The latter can be explained by the subsequent EPSP-synthase reaction, taking S3P and PEP for (reversible) EPSP synthesis. Corresponding $K_{\mathrm{m}}$ values of $10-20 \mu \mathrm{M}$ for PEP and 2.5$3.6 \mu \mathrm{M}$ for $\mathrm{S} 3 \mathrm{P}$ were measured by in vitro experiments (see Table 1), giving rise to the assumption that the decreasing PEP levels could hamper EPSP-synthase activity in vivo.

To explain the high 3-DHS and 3-DHQ dependence on $\mathrm{P} 5 \mathrm{P}$, consideration should be given to their proximity to 
the $\mathrm{P} 5 \mathrm{P}$ stimulation, the missing $\mathrm{DAH}(\mathrm{P})$ buffer, and the close connection between the 3-DHQ and 3-DHS pools. However, the question arises why the PEP reliance of the DAHP synthase, the 3-DHQ "supplier", led to only surprisingly small correlation values. In wild-type $E$. coli cells, almost $80 \%$ of the total DAHP synthase activity is realized via AroG and to a less extent by AroF. The tryptophan sensitive AroH is only of minor importance (Pittard, 1996). Because strain B possessed a plasmidencoded $a r o F^{f b r}$ overexpression, it can be expected that AroF ${ }^{\mathrm{tbr}}$ together with AroG were responsible for the prevailing amount of carbon flux into the aromatic amino acid pathway. As indicated in Table 1, a high PEP affinity of native $\operatorname{AroF}\left(K_{\mathrm{m}} \sim 13 \mu \mathrm{M}\right)$ and some conflicting results with respect to AroG $\left(K_{\mathrm{m}} \sim 5-80 \mu \mathrm{M}\right)$ were found by in vitro studies. Provided that the feedback-resistant $\mathrm{AroF}^{\mathrm{fbr}}$ mutant possessed similar PEP affinity compared to AroF (Jossek et al., 2001), PEP-limited AroF ${ }^{\mathrm{fbr}}$ or AroG activities should not necessarily occur during the first phase of experimental observations when intracellular PEP levels were sufficiently high. However, they were likely to occur during the later phase, when intracellular PEP levels decreased.

With respect to Figure 4 (strain B), the remarkable observation is made that all precursors and intermediates show a zero-crossing at about $17.5 \mathrm{~s}$, indicating the start of a common in-phase oscillation after a short pool inertia. At this time, intracellular PEP levels had already diminished to about $35 \mu \mathrm{M}$. This was the motivation for a separate correlation analysis using all pool velocity data from $17.5 \mathrm{~s}$ onward. We found that all intermediates showed very high correlations to PEP (3-DHQ, 0.87; 3-DHS, 0.87; SHI, 0.96; S3P, 0.96) and only low correlations to P5P (up to 0.37 in the case of 3-DHQ). All intermediates were now closely intercorrelated, showing values of not less than 0.74 in subsequent reactions. From this we concluded that DAHP-synthase activity (meaning AroF ${ }^{\mathrm{tbr}}$, AroG, and to a minor extent $\mathrm{AroH}$ ) obviously became PEP-limited, thus switching the former dependence on P5P to PEP, which is expressed by the corresponding correlation change of 3-DHQ and 3-DHS. Pool velocity oscillations of these compounds were now inphase with SHI and S3P, which is in contrast to the previous situation. As a consequence, oscillation ringing occurs, as seen in the pool velocity data of $3-\mathrm{DHQ}$ and 3-DHS.

The finding that limited PEP supply dominated pool velocity oscillations in the aromatic amino acid pathwayand especially those of S3P, the substrate of EPSPsynthase-can also be understood as an indication for further production-strain optimization. Previous studies of Draths et al. (1992), Patnaik and Liao (1994), Patnaik et al. (1995), Berry (1996), Flores et al. (1996), and Li et al. (1999) already addressed the problem of well-balanced PEP and E4P availability to achieve a maximum carbon flux into the aromatic amino acid pathway. It has also been shown by Karutz et al. (1998) that an optimized precursor supply can be achieved by a combination of transketolase (tktA) overexpression together with a phosphoenolpyruvate synthase (pps) amplification or by implementing an alternative, non-pts-based glucose uptake. In general, our results support these findings. However, with respect to the correlation analysis of an L-phenylalanine producer, PEP availability is assigned to be even of higher importance than E4P supply, expressed by P5P.

Finally, the pool efflux capacity criterion was applied again (Figure 5), on the basis of the pool velocities presented in Figure 4 (strain B). Absolute minima like 1.24 (3-DHQ), 0.92 (3-DHS), 0.37 (SHI), and 0.3 (S3P) were calculated. As a consequence, AroB, AroD, and AroE, using $\mathrm{DAH}(\mathrm{P}), 3-\mathrm{DHQ}$, and 3-DHS as substrates, could be excluded as potential candidates for metabolic engineering; i.e., they are expected to exert only minor flux control. Because the observed criterion differences for SHI and S3P were relatively small, AroL/AroK (shikimate kinases), catalyzing SHI to S3P, as well as AroA (EPSP synthase), catalyzing S3P with PEP to EPSP, were targets for further metabolic engineering. In the case of AroK/AroL, aroL should be preferred, owing to its higher shikimate affinity (see Table 1). Again, this target identification is in agreement with the previous observations of Dell and Frost (1993) and Snell et al. (1995). The criterion even succeeded to avoid the misleading identification of AroE (shikimate dehydrogenase) as a target for gene overexpression, which could occur if pure concentration analysis would have been performed (Dell and Frost, 1993; Snell et al., 1995). Nevertheless, the simple pool efflux capacity criterion should be used with care, as its applicability should be limited to linear pathways and superimposing effects, such as additional substrate limitations of bimolecular enzyme kinetics, are not thoroughly considered.

\section{Conclusion}

The results show that the concerted approach of glucose pulse, rapid sampling, metabolic profiling, data analysis, and strain construction has successfully been applied to stimulate and analyze dynamics in an anabolic pathway, studying the L-phenylalanine production in $E$. coli as an example. Potential drawbacks, such as limited analytical access or signal dilution, have been overcome by using knock-out mutants, standard purification, LCMS/MS analysis, and fortification of the carbon flux into the aromatic amino acid pathway expressing plasmidencoded genes such as $a r o F^{f b r}$ (and $a r o B$ ).

On the basis of the dynamic metabolite curves, the standardized pool velocities were calculated. Thereof, the maximum (negative) values of each species were identified and ranked, thus defining the simple, data-driven pool efflux capacity (PEC) criterion for a straightforward, easy-to-use system analysis. As a consequence, reaction steps exerting significant flux control have been experimentally reconfirmed (for example, 3-DHQ-synthase in strain A). The repeated application of PEC with the $a r o B$ overexpressed strain $\mathrm{B}$ permitted the conclusion that aro $D$ and $a r o E$ are not necessarily metabolic engineering targets, but aroL and aroA are. In addition, the correlation analysis of the oscillating standardized pool velocity curves allowed the reconstruction of the aromatic amino acid reaction sequence. Beyond it, it was found that PEP supply should preferably be optimized for production strain improvement.

The experimental and analytical approach is not exclusive to the analysis of the aromatic amino acid pathway. In contrast, considering the fact that a positive signal stimulation (rising precursor concentration) should be favored to ensure measurable pool changes in the pathway, the example of negative PEP signaling into the aromatic amino acid pathway anticipates even higher signals in positively stimulated routes. This includes, for instance, amino acids, vitamins, or antibiotics originating from precursors such as pyruvate, intermediates of the pentose phosphate pathway, or even TCA metabolites such as oxaloacetate, for which positive glucose pulse responses were already found (data not shown).

The analysis of pathway dynamics offers new insights because of the high data density gained after stepfunction or Dirac-like system stimulation. Because short- 
term observation windows were chosen, gene regulatory effects can be excluded, thus focusing the analysis on pure enzyme kinetics. Therefore, corresponding dynamic data represent a valuable basis for model-based in vivo enzyme kinetic identification or thorough oscillation analysis, as is currently being performed. It can be assumed that model identification of the linear pathway will be simplified compared to complex network analysis, because cross-interactions usually encountered should not be expected.

Indeed, no detailed model analysis was necessary to obtain the presented results. Pathway mechanisms were elucidated by simple correlation and oscillation analysis, and even the pathway reconstruction is enabled by correlation analysis, too. Hence, the analysis of pathway dynamics stimulated by glucose pulses should no longer be restricted to central metabolism; rather, it should be extended to anabolic pathways. This enables the use of simple criteria, such as the pool efflux capacity criterion to visualize the distributed flux control in a reaction sequence alternative to classical MCA approaches.

\section{Acknowledgment}

The authors would like to thank Prof. Wandrey for his generous support and the excellent working conditions provided in the Institute of Biotechnology at Forschungszentrum Jülich $\mathrm{GmbH}$. The authors are indebted to Dr. M. Krämer, Dr. J. Bongaerts (both at DSM Biotech GmbH, Jülich), and U. Degner (Institute of Biotechnology) for constructing L-phenylalanine producers and knock-out mutants, as presented in the article, and Prof. H. Vereecken and Dr. H. -D. Narres (Institute of Agrosphere, Forschungszentrum Jülich $\mathrm{GmbH}$ ) for their invaluable cooperation in the LC-MS field. Thanks are also due to Prof. W. Wiechert (University of Siegen) and Prof. H. Sahm (Institute of Biotechnology) for their fruitful discussions during the preparation of this article. We would also like to thank our cooperating partners at DSM, The Netherlands, and at DSM Biotech GmbH, Jülich, Germany, for their valuable cooperation in the FAME project (BMBF, grant 0311644), which represents the notional frame of the results presented here. The project was partially funded by the Deutsche Forschungsgemeinschaft DFG (TA 241/2-1 and TA 241/2-2).

\section{Notation}

3-DHQ 3-dehydroquinate

3-DHS 3-dehydroshikimate

AroA EPSP synthase

AroB 3-DHQ synthase

AroD 3-DHQ dehydratase

AroE shikimate dehydrogenase

AroF DAHP synthase, tyr-sensitive

AroG DAHP synthase, phe-sensitive

AroH DAHP synthase, trp-sensitive

AroK shikimate kinase I

AroL shikimate kinase II

DAH 3-deoxy-D-arabino-heptulosonate

DAHP 3-deoxy-D-arabino-heptulosonate 7-phosphate

E4P erythrose 4-phosphate

EPSP 5-enolpyruvoylshikimate 3-phosphate

G6P glucose 6-phosphate

L-Phe L-phenylalanine

L-Trp L-tryptophan

L-Tyr L-tyrosine

P5P lumped pentose phosphate pool
PEP phosphoenolpyruvate

pts phosphoenolpyruvate:carbohydrate phosphotransferase system

PYR pyruvate

S3P shikimate 3-phosphate

SHI shikimate

tca tricarboxylic acid cycle

\section{References and Notes}

Akowski, J. P.; Bauerle, R. Steady-state kinetics and inhibitor binding of 3-deoxy-D-arabino-heptulosonate-7-phosphate synthase (tryptophan sensitive) from Escherichia coli. Biochemistry 1997, 36, 15817-15822.

Bader, A. A systematic approach to standard addition methods in instrumental analysis. J. Chem. Edu. 1980, 57 (10), 703706.

Bergmeyer, H. Methods of Enzymatic Analysis, 3rd ed.; Verlag Chemie: Weinheim, 1985; Vols. 6 and 7.

Berry, A. Improving production of aromatic compounds in Escherichia coli by metabolic engineering. TIBTECH 1996 , 14, 250-256.

Bongaerts, J.; Krämer, M.; Müller, U.; Raeven, L.; Wubbolts, M. Metabolic Engineering for Microbial Production of Aromatic Amino Acids and Derived Compounds. Metab. Eng. 2001, 3, 289-300.

Buchholz, A.; Takors, R.; Wandrey, C. Quantification of Intracellular Metabolites in Escherichia coli K12 Using Liquid Chromatographic-Electrospray Ionization Tandem Masss Spectrometric Techniques. Anal. Biochem. 2001, 295, 129137.

Chassagnole, C.; Noisommit-Rizzi, N.; Schmid, J. W.; Mauch, K.; Reuss, M. Dynamic Modelling of the Central Carbon Metabolism of Escherichia coli. Biotechnol. Bioeng. 2002, 79 (3), 53-73.

Chaudhuri, S.; Duncan, K.; Coggins, J. R. 3-Dehydroquinate dehydratase from Escherichia coli. Methods Enzymol. 1987, $142,320-324$.

Chaudhuri, S.; Lambert, J. M.; McColl, L. A.; Coggins, J. R. Purification and characterization of 3-dehydroquinase from Escherichia coli. Biochem. J. 1986, 239, 699-704.

Coggins, J. R.; Boocock, M. R.; Chaudhuri, S.; Lambert, J. M.; Lumsden, J.; Nimmo, G. A.; Smith, D. D. S. The arom Multifunctional Enzyme from Neurospora crassa. Methods Eynzmol. 1987, 142, 325.

De Feyter, R. Shikimate kinases from Escherichia coli K12. Methods Enzymol. 1987, 142, 355-361 (review).

De Feyter, R. C.; Pittard, J Purification and properties of shikimate kinase II from Escherichia coli K-12. J. Bacteriol. 1986, 165, 331-333.

Degenring, D.; Froemel, C.; Dikta, G.; Takors, R. Sensitivity analysis for the reduction of complex metabolism models. $J$. Process Control 2004, 14, 729-745.

Dell, K. A.; Frost, J. W. Identification and Removal of Impediments to Biocatalytic Synthesis of Aromatics from D-Glucose: Rate-Limiting Enzymes in the Common Pathway of Aromativ Amino Acid Biosynthesis. J. Am. Chem. Soc. 1993, 115, 11581-11589.

Draths, K. M.; Pompliano, D. L.; Conley, D. L.; Frost, J. W.; Berry, A.; Disbrow, G. L.; Staversky, R, J.; Levense, J. C. Biocatalytic Synthesis of Aromatics from D-Glucose: The Role of Transketolase. J. Am. Chem. Soc. 1992 114, 3956-3962.

Duncan, K.; Lewendon, A.; Coggins, J. R. The purification of 5-enolpyruvoylshikimate 3-phosphate synthase from an overproducing strain of Escherichia coli. FEBS Lett. 1984, 165, 121-127.

Fiehn, O. Combining genomics, metabolome analysis and biochemical modelling to understand metabolic networks. Comp. Funct. Genom. 2001, 2, 155-168.

Flores, N.; Xiao, J.; Berry, A.; Bolivar, F.; Valle, F. pathway engineering for the production of aromatic compounds in Escherichia coli. Nature Biotechnol. 1996, 14, 620-623. 
Frost, J. W.; Lievense, J. Prospects for biocatalytic synthesis of aromatics in the 21 st century. New J. Chem. 1993, 18, 341348.

Fürste, J. P.; Pansegrau, W.; Frank, R.; Blöcker, H.; Scholz, P.; Bagdasarian, M.; Lanka, E. Molecular Cloning of the Plasmid RP4 Primase Region in a multi-host-range tacP Expression Vector. Gene 1986, 48, 119-131.

Gerigk, M. R.; Maass, D.; Kreutzer, A.; Sprenger, G.; Bongaerts, J.; Wubbolts, M.; Takors, R. Enhanced pilot-scale fed-batch L-phenylalanine production with recombinant Escherichia coli by fully integrated reactive extraction. Bioproc. Biosyst. Eng. 2002a, 25, 43-52.

Gerigk, K.; Bujnicki, R.; Ganpo-Nkwenkwa, E.; Bongaerts, J.; Sprenger, G.; Takors, R, Process Control for Enhanced l-Phenylalanine Production Using Different Recombinant E. coli Strains, Biotechnol. Bioeng. 2002b 80 (7), 746-754.

Grewe, R.; Jeschke, J. P. Die Synthese der 5-Dehydrochinsäure. Ber. Dtsch. Chem. Ges. 1956, 89, 2080-2088.

Gruys, K. J.; Walker, M. C.; Sikorski, J. Substrate synergism and the steady-state kinetic reaction mechanism for EPSP synthase from Escherichia coli. Biochemistry 1992, 31, 55345544.

Hurlebaus, J.; Buchholz, A.; Alt, W.; Wiechert, W.; Takors, R. MMT-A Pathway Modeling Tool for Data from Rapid Sampling Experiments. Silico Biol. 2002, 2, 467-484.

Jossek, R.; Bongaerts, J.; Sprenger, G. A. Characterization of a new feedback-resistant 3-deoxy-D-arabino-heptulosonate 7-phosphate synthase AroF of Escherichia coli. FEMS Microbiol. Lett. 2001, 202, 145-148.

Karutz, M.; Kraemer, M.; Sahm, H.; Sprenger, G. Microbial preparation of substances from aromatic metabolism I. WO98/ 18936. Patent granted in the USA (6316232), ZA (9568/97) and pending for EP, CN, JP, KR, and DE, 1998.

Kleanthous, C.; Deka, R.; Davis, K.; Kelly, S. M.; Cooper, A.; Harding, S. E.; Price, N. C.; Hawkins, A. R.; Coggins, J. R. A comparison of the enzymological and biophysical properties of two distinct classes of dehydroquinase enzymes. Biochem. J. 1992, 282, 687-695.

Lange, H. C.; Eman, M.; van Zuijlen, G.; Visser, D.; van Dam, J. C.; Frank, J.; Teixeira de Mattos, M. J.; Heijnen, J. J. Improved rapid sampling for in vivo kinetics of intracellular metabolites in Saccharomyces cerevisiae. Biotechnol. Bioeng. 2001, 75 (4), 406-415.

Lemarechal, P.; Froussios, C. Level, M.; et al. Interaction of phosphonate and homophosphonate analogues of 3-deoxy-Darabino heptulosonate 7-phosphate with 3-Dehydroquinate synthetase from Escherichia coli. Biochem Bioph Res. 1980 , 92 (4), 1104-1109.

Lewendon, A.; Coggins, J. R. 3-Phosphoshikimate 1-carboxyvinyltransferase from Escherichia coli. Methods Enzymol. 1987, $142,342-348$

Li, K.; Mikola, M. R.; Draths, K. M.; Worden, R. M.; Frost, J. W. Fed-Batch Fermentor Synthesis of 3-Dehydroshikimic Acid Using Recombinant Escherichia coli. Biotechnol. Bioeng. 1999, 64(1), 61-73.

Maitra U. S.; Sprinson, D. B. 5-Dehydro-3-deoxy-D-arabinoheptulosonic acid 7-phosphate. J. Biol. Chem. 1978, 253(15), 5426-5430.

Mashego, M. R.; Wu, L.; Van Dam, J. C.; Ras, C.; Vinke, J. L.; Van Winden, W. A.; Van Gulik, W. M.; Heijnen, J. J. MIRACLE: Mass Isotopomer Ratio Analysis of U-13CLabeled Extract. A New Method for Accurate Quantification of Changes in Concentrations of Intracellular Metabolites. Biotechnol. Bioeng. 2004, 85 (6), 620-628.

Mauch, K.; Vaseghi, S.; Reuss, M. 2000 Quantitative Analysis of Metabolic and Signaling Pathways in Saccharomyces cerevisiae. In Bioreaction Engineering; Schügerl, K, Bellgardt, K. H., Eds; Springer-Verlag: Heidelberg, ISBN 3-540-66906$\mathrm{X}$.

McCandliss, R. J.; Poling, M. D.; Hermmann, K. M. 3-DeoxyD-arabino-heptulusonate 7-phosphate synthase-Purification and molecular characterization of phenylalanine-sensitive isoenzyme from Escherichia coli. J. Biol. Chem. 1978, 253 (12), 4259-4265.
Mehdi, S.; Frost, J. W.; Knowles, J. Dehydroquinate Synthase from Escherichia coli and its Substrate 3-Deoxy-D-arabinoheptulosonic Acid 7-phosphate. Methods Enzymol. 1987, 142, 306-314.

Oldiges, M.; Takors, R. Applying Metabolic Profiling Techniques for Stimulus-Response Experiments: Chances and Pitfalls. Adv. Biochem. Eng. Biotechnol. 2004, in press.

Ostergaard, S.; Olsson, L.; Nielsen, J. In vivo dynamics of galactose metabolism in Saccharomyces cerevisiae: Metabolic fluxes and metabolite levels. Biotechnol. Bioeng. 2001, 73 (5), $412-425$.

Patnaik, R.; Liao, J. C. Engineering of Escherichia coli Central Metabolism for Aromatic Metabolite Production with Near Theoretical Yield. Appl. Environ. Microbiol. 1994, 10, 39033908.

Patnaik, R.; Spitzer, R. G.; Liao, J. C. Pathway Engineering for Production of Aromatics in Escherichia coli: Confirmation of Stoichiometric Analysis by Independent Modulation of AroG, TktA and Pps Activities. Biotechnol. Bioeng. 1995, 46, 361-370.

Pittard, A. J. Biosynthesis of the Aromatic Amino Acids. In Escherichia coli and Salmonella typhimurium-Cellular and Molecular Biology; Neidhardt, F. C., Ed.; ASM press: Washington, DC, 1996.

Ramilo, C. A.; Evans, J. N. S. Overexpression, purification, and characterization of tyrosine-sensitive 3-deoxy-D-arabino-heptulosonic acid 7-phosphate synthase from Escherichia coli. Protein Expr. Purif. 1997, 9, 253-261.

Rizzi, M.; Baltes, M.; Theobald, U.; Reuss, M. In vivo Analysis of Metabolic Dynamics in Saccharomyces cerevisiae: II Mathematical Model. Biotechnol. Bioeng. 1997, 55 (4), 592608.

Rüffer, N.; Heidersdorf, U.; Kretzers, I.; Sprenger, G. A.; Raeven, L.; Takors, R. Fed-batch L-Phenylalanine Production with recombinant E. coli using Liquid-Liquid Centrifuges for Integrated Product Separation and Concentration. Bioprocess Biosys. Eng. 2004, 26, 239-248.

Rujiter, G. J. G.; Visser, J. Characterisation of Aspergillus niger phosphoglucose isomerase use for quantitative determination of erythrose 4-phosphate. Biochimie 1999, 81, 1 (3), 267-272.

Sauter, T.; Kremling, A.; Bettenbrock, K.; Fischer, S.; Gilles, E. D. Analysis of the dynamics of the Escherichia coli PTS in different time windows. In Proceedings of the $5^{\text {th }}$ German Workshop on Artificial Life; Polani, D., Kim, J., Martinek, T., Eds; IOS Press: Lübeck, Germany, 2002; ISBN 3-89838030-Aka.

Schäfer, U.; Boos, W.; Takors, R.; Weuster-Botz, Automated Sampling Device for Monitoring Intracellular Metabolite Dynamics. Anal. Biochem. 1999, 270, 88-96.

Schmid, A.; Dordick, J. S.; Hauer, B.; Kiener, A.; Wubbolts, M.; Witholt, B. Industrial biocatalysis today and tomorrow. Nature 2001, 409, 258-268.

Schmitz, M.; Hirsch, E.; Bongaerts, J.; Takors, R. Pulse Experiments as a Prerequisite for the Quantification of In Vivo Enzyme Kinetics in Aromatic Amino Acid Pathway of Escherichia coli. Biotechnol. Prog. 2002, 18, 935-941.

Schoner, R.; Herrmann, K. M 3-Deoxy-D-arabino-heptulosonate 7-phosphate synthase. Purification, properties, and kinetics of the tyrosine-sensitive isoenzyme from Escherichia coli. J. Biol. Chem. 1976, 251, 5440-5447.

Simmons, S. The metabolism of Phenylalanine and Tyrosine in Mutant Strains of Escherichia coli. J. Biol. Chem. 1950, 185, $755-762$.

Simpson, R. J.; Davidson, B. E. Studies on 3-deoxy-D-arabinoheptulosonate-7-phosphate synthetase(phe) from Escherichia coli K12. 2. Kinetic properties. Eur. J. Biochem. 1976, 70, 509-516.

Snell, K. D.; Draths, K. M.; Frost, J. W. Synthetic Modification of Escherichia coli Chromosome: Enhancing the Biocatalytic Conversion of Glucose into Aromatic Chemicals. J. Am. Chem. Soc. 1996, 118, 5605-5614.

Theobald, U.; Mailinger, W.; Baltes, M.; Rizzi, M.; Reuss, M. In vivo Analysis of Metabolic Dynamics in Saccharomyces cerevisiae: I. Experimental Observations. Biotechnol. Bioeng. 1997, 55 (2), 305-316. 
Van Dam, J. C.; Eman, M. R.; Frank, J.; Lange, H. C.; van Dedem, G. W. K.; Heijnen, J. J. Analysis of glycolytic intermediates in Saccharomyces cerevisiae using anion exchange chromatography and electrospray ionization with tandem mass spectrometric detection. Anal. Chim. Acta 2002 460, 209-218.

Vaseghi, S.; Baumeister, A.; Rizzi, M.; Reuss, M. In vivo Dynamics of the Pentose Phosphate Pathway in Saccharomyces cerevisiae. Metab. Eng. 1999, 1, 128-140.

Visser, D.; Heijnen, J. J. Dynamic simulation and metabolic redesign of a branched pathway using linlog kinetics. Metab. Eng. 2003, 5, 164-176.

Westerhoff, H. V. The Silicon Cell, Not Dead but Live! Metab. Eng. 2001, 3, 207-210.

Westerhoff, H. V.; Kell, D. B. What biotechnologists knew all along...? J. Theor. Biol. 1996, 182, 411-420.

Weuster-Botz, D. Sampling Tube Device for Monitoring Intracellular Metabolite Dynamics. Anal. Biochem. 1997, 246, $225-233$.

Weuster-Botz, D.; de Graaf, A. A. Reaction engineering methods to study intracellular metabolite concentrations. Adv. Biochem. Eng. Biotechnol. 1996, 54, 76-108.
Widlanski, T.; Bender, S. L.; Knowles, P. F. Dehyroquinate synthase-a sheep in wolfs clothing. Am. Chem. Soc. 1989, 111, 2299-2300.

Williams, J. F.; Blackmore, P. F.; Duke, C. C.; MacLeod, J. K. Fact, uncertainty and speculation concerning biochemistry of d-erythrose-4-phosphate and its metabolic roles. Int. J. Biochem. 1980, 12, 339-344.

Yi, J.; Li, K.; Draths, K. M.; Frost, J. W. Modulation of Phosphoenolpyruvate Synthase Expression Increases Shikimate Pathway Product Yields in E. coli. Biotechnol. Prog. 2002, 18, 1141-1148.

Zeppenfeld, T.; Larisch, C.; Lengeler, J. W.; Jahreis, K. Glucose Transporter Mutants of Escherichia coli K-12 with Changes in Substrate Recognition of IICB ${ }^{\text {Glc }}$ and Induction Behaviour of the ptsG Gene. J. Bacteriol. 2000, 182, 4443.

Accepted for publication August 26, 2004

BP0498746 